\title{
LA OFERTA DE EDUCACIÓN SUPERIOR DE PREGRADO EN CHILE DESDE LA PERSPECTIVA TERRITORIAL: INEQUIDADES Y ASIMETRÍAS EN EL MERCADO*
}

\author{
Sebastián Donoso, Óscar Arias, Macarena Weason, \\ Claudio Frites ${ }^{1}$
}

\begin{abstract}
RESUMEN
El artículo tiene por finalidad analizar la distribución de la educación superior desde una perspectiva territorial, a partir de los datos de las vacantes en todos los programas académicos del año 2011. Para estos efectos, se analizan las vacantes existentes en cada tipo de institución, en cada área del conocimiento y tipo de carrera. Con estos datos - de región y comuna- se revisa la oferta en términos de su complejidad y formas de distribución, estableciéndose una tipología que las describe combinada con el componente territorial. Se presentan las consideraciones finales acerca de la evolución de la oferta en educación superior, destacando la debilidad del mercado para abordar las políticas de desarrollo del capital humano en forma sinérgica con las estrategias de mejora y aptitudes de los territorios.
\end{abstract}

Palabras clave: educación superior, regiones, territorio, inequidad, Chile

\section{UNDERGRADUATE HIGHER EDUCATION SUPPLY IN CHILE FROM A TERRITORIAL PERSPECTIVE: INEQUITIES AND ASYMMETRIES IN THE MARKET}

\begin{abstract}
This article is aimed to analyze the distribution of higher education from a territorial perspective based on data about vacant places in all academic programs of year 2011. To this end, it examines vacant places available in each type of institution, in each area of knowledge and type of career. With this data, about region and municipality, it reviews supply in terms of complexity and forms of distribution, setting a typology that describes them along with the territorial component. It presents final considerations about the evolution of the supply in higher education and highlights the market weaknesses to address development policies on human capital in synergy with improvement strategies and territorial aptitudes.
\end{abstract}

Keywords: higher education, regions, territory, inequity, Chile

*Proyecto financiado por la Dirección de Investigación de la Universidad de Talca, Di 006050.

1 Equipo de investigación del Instituto de Investigación y Desarrollo Educacional de la Universidad de Talca, Talca, Chile. Contacto: sdonoso@utalca.cl 


\section{LA OFERTA DE EDUCACIÓN SUPERIOR DE PREGRADO EN CHILE DESDE LA PERSPECTIVA TERRITORIAL: INEQUIDADES Y ASIMETRÍAS EN EL MERCADO}

\section{Antecedentes}

Las características del sistema de educación superior chileno a partir del año 1980 -hito que marca la reforma estructural al sistema universitario- hasta la fecha se sustentan en una baja regulación acompañada de una fuerte privatización del sector, factores claves que han significado una explosiva expansión de vacantes e instituciones, la cual solamente en los últimos veinte años ha implicado cuadruplicar su tamaño (CNED, 2011:7). No obstante esta constatación, desde la perspectiva territorial este proceso ha implicado la provisión desigual de la oferta formativa de este nivel del sistema, materia que se estima difícil de corregir, sin que medie una política pública que corrija el mercado y sustente opciones formativas más convergentes con los ejes de desarrollo territorial del país y de las regiones en una visión de mediano y largo plazo.

El trabajo analiza la oferta regional de educación superior de pregrado en Chile, a partir de los datos de vacantes en todos los programas académicos del país, consignados en la base estadística Índices del Consejo Nacional de Educación (CNED) del año 2011, con el fin de tener una visión actualizada de la realidad regional respecto a educación postsecundaria ${ }^{2}$ y de esta forma relacionar la formación de capital humano avanzado (KH) con el desarrollo -en su sentido más completo- de los territorios.

El eje del análisis son las formas que asume la distribución de la oferta en educación superior en las regiones y comunas, que se

2 En la versión revisada, esta base no contaba con los datos de vacantes para la Universidad del Biobío y de la Universidad de Atacama, los que fueron extraídos desde la información publicada por estas mismas instituciones. 
definen como las unidades de análisis del estudio. La hipótesis de trabajo es que debido a la inequidad socioeconómica y territorial que caracteriza al país dicha oferta sustentada en el mercado refleja esta condición, distribuyéndose de manera desigual, tanto entre regiones como al interior de cada una de ellas. La consideración de la oferta de vacantes reside en el hecho de que no existe información pública suficiente respecto de las matrículas efectivas por institución; adicionalmente se sabe que la fijación de vacantes tiene un referencial respecto del contexto en que se sitúa, al menos en lo que se refiere a densidad poblacional, en razón de lo cual, se la utiliza como variable proxy de demanda efectiva.

El estudio se ordena en cuatro partes. Primero se revisan los datos de vacantes según cada tipo de institución: centros de formación técnica (CFT), institutos profesionales (IP) y universidades (UES). Estas últimas se subdividen en tres categorías: las pertenecientes al Estado (UES-E), privadas tradicionales (UES-P) -existentes antes de 1980-y privadas no tradicionales (UES-PP) -creadas con posterioridad a 1980-. En segundo lugar se establecen las vacantes en cada área del conocimiento ${ }^{3}$, analizándolas según tipo de carrera: bachillerato, licenciatura, plan común, profesional, profesional con licenciatura y técnico de nivel superior. En tercer lugar, a partir de la distribución regional y comunal de esos datos se revisa la complejidad de la oferta y sus formas de distribución, según: volumen, diversidad y niveles de formación. Por último, se presentan las conclusiones y algunas preguntas e ideas en torno a la evolución de la oferta en educación terciaria.

\section{Descripción general de la oferta de vacantes por regiones}

Una primera mirada a la oferta de educación superior regional da cuenta de una realidad que teniendo ciertos puntos de convergencia es esencialmente muy heterogénea. Ello por cuanto en todas las regiones (el país se organiza en quince) hay presencia de este nivel

3 Las áreas se ajustan a la clasificación UNESCO, no obstante hay ciertas diferencias con el objeto de permitir un análisis más detallado. 
educacional; sin embargo, la oferta de carreras e instituciones varía sustancialmente según la densidad poblacional de los territorios y el desarrollo socioeconómico presente y sus proyecciones al respecto.

La cobertura territorial de la educación superior en el país alcanzó a 72 de las 345 comunas, lo que representa un 21\% del total del territorio, esto evidencia una importante concentración en esta materia. No obstante, efectivamente se presenta una diversidad relevante entre regiones (tabla $n .{ }^{\circ} 1$, figura $n . .^{\circ} 1$ ), situación que es radicalmente diferente al comportamiento de la oferta de enseñanza primaria y secundaria, cuya cobertura y dispersión territorial es muy superior a la oferta de educación postsecundaria por una serie de consideraciones históricas, económicas y sociales que en la actualidad se identifican con cobertura y financiamiento universal por parte del Estado y con la condición de obligatoriedad de provisión que el mismo se autoimpone respecto de la enseñanza primaria y secundaria.

Con relación a la oferta comunal de educación superior, la Región Metropolitana (donde se sitúa la capital del país) reúne el 32\% de la oferta territorial (23 comunas), en su mayoría dispuestas en el área metropolitana urbana de la ciudad de Santiago. Le siguen las regiones de Valparaíso, del Biobío, del Maule y de La Araucanía, con una oferta que varía entre 9 y 6 comunas, representando del 12,5\% al $8,3 \%$ del total. Finalmente, las restantes regiones no tienen más de tres comunas con oferta en educación terciaria, destacando en este último grupo los territorios extremos del norte del país: Arica/Parinacota, Tarapacá, y del sur, Aysén, con solamente una comuna con oferta en la región, lo que corresponde al 1,4\% del total de comunas con este tipo de educación, materia que se relaciona con la alta concentración de población del territorio. 
Figura n. ${ }^{\circ}$ 1. Distribución regional de las vacantes en ES y tasa de vacantes respecto de la población de 20 a 24 años.

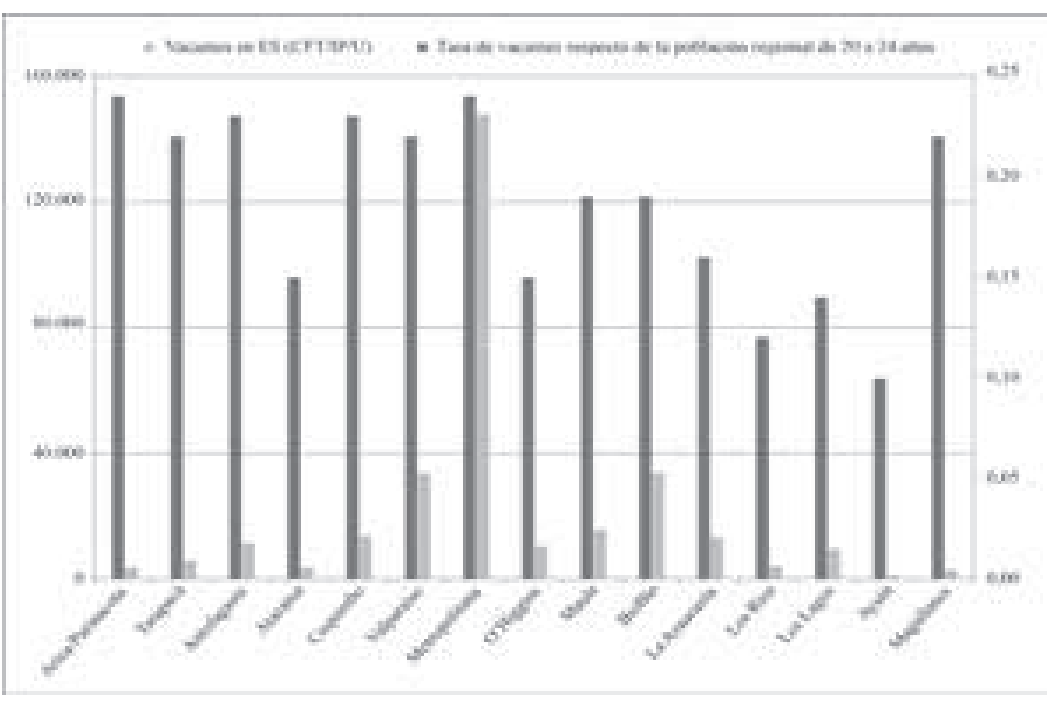

Fuente: CNED (2011).

Tabla n. ${ }^{\circ}$ 1. Comunas con oferta de educación superior en las regiones.

\begin{tabular}{|l|c|c|c|c|}
\hline Región & $\begin{array}{c}\text { N. }{ }^{\circ} \text { de } \\
\text { comunas con } \\
\text { oferta de ES }\end{array}$ & $\begin{array}{c}\text { \% comunas con oferta de ES } \\
\text { respecto del total comunas } \\
\text { con oferta de ES país (72) }\end{array}$ & $\begin{array}{c}\text { Total } \\
\text { comunas de } \\
\text { la región }\end{array}$ & $\begin{array}{c}\text { \% comunas con oferta } \\
\text { de ES respecto al total de } \\
\text { comunas de la región }\end{array}$ \\
\hline Arica/Parinacota & 1 & 1,4 & 4 & 25,0 \\
\hline Tarapacá & 1 & 1,4 & 7 & 14,3 \\
\hline Antofagasta & 2 & 2,8 & 9 & 22,2 \\
\hline Atacama & 2 & 2,8 & 9 & 22,2 \\
\hline Coquimbo & 3 & 4,2 & 15 & 20,0 \\
\hline Valparaíso & 9 & 12,5 & 38 & 23,7 \\
\hline Metropolitana & 23 & 31,9 & 52 & 44,2 \\
\hline O'Higgins & 3 & 4,2 & 33 & 9,1 \\
\hline Maule & 6 & 8,3 & 30 & 20,0 \\
\hline Biobío & 7 & 9,7 & 54 & 13,0 \\
\hline La Araucanía & 6 & 8,3 & 32 & 18,8 \\
\hline Los Ríos & 2 & 2,8 & 12 & 16,7 \\
\hline Los Lagos & 3 & 4,2 & 30 & 10,0 \\
\hline Aysén & 1 & 1,4 & 10 & 10,0 \\
\hline Magallanes & 3 & 4,2 & 11 & 27,3 \\
\hline TOTAL & 72 & 100 & 346 & 20,8 \\
\hline
\end{tabular}

Fuente: CNED (2011). 

PERSPECTIVA TERRITORIAL: INEQUIDADES Y ASIMETRÍAS EN EL MERCADO - S. DonOSO, Ó. Arias, M. Weason, C. Frites

Complementariamente, se puede identificar el peso que tienen las comunas con educación superior respecto al total de las comunas de cada región, lo que representa un primer indicador respecto de cómo se distribuye la oferta al interior de cada una de las regiones. Con menor representación respecto al total de comunas de la región, O’Higgins, Los Lagos y Aysén están entre las que no superan el 10\% de la región. No obstante, cada uno de estos casos es diferente. En la Región de O'Higgins la gran influencia y conectividad con Santiago explica de manera dominante esta situación ${ }^{4}$. Por su parte, la Región de Los Lagos posee elevados niveles de población rural, asociado a una baja densidad poblacional con dificultades climáticas y de conectividad, que inciden en el desplazamiento cotidiano de su población. La Región de Aysén es el caso más crítico, su población total no supera los 100 mil habitantes (INE, 2012), tiene un gran territorio, baja densidad poblacional y elevada concentración de población en dos de las diez comunas de la región, además del clima riguroso y severos problemas de conectividad terrestre de sus habitantes.

En el extremo opuesto a lo descrito, la Región Metropolitana destaca sobre todas las regiones con una representación del 44,2\%, con una gran densidad poblacional, con alta conectividad de su población, el PIB mayor del país y los ingresos económicos más altos (BC, 2012).

A continuación, en la tabla $n .^{\circ} 2$ se da cuenta de la oferta territorial de vacantes (año 2011), determinándose 310758 cupos para ingresar a la educación superior. La media de vacantes por región correspondió a 20717 , aunque la dispersión al respecto es alta pues la mitad de las comunas ofreció hasta 10422 vacantes, siendo por tanto un estimador muy débil.

Consistente con lo expuesto, la Región Metropolitana registra la mayor cantidad de vacantes, 147848 , equivalentes al 47,6\% de la oferta nacional, seguidas por las regiones de Valparaíso con 33801 y Biobío con 34 006, totalizando entre ambas el 21,8\% de la oferta

4 Son escasos los proyectos de educación superior exitosos en esa región en los últimos 30 años, pese a muchos esfuerzos los fracasos han sido muy frecuentes por la influencia de la ciudad de Santiago y de la Región Metropolitana como polo de atracción para los estudiantes. 
nacional de vacantes. Luego se sitúan el conjunto de regiones que controlan individualmente entre un 5\% y un 3\% de las vacantes totales: Antofagasta, Coquimbo, O’Higgins, Maule y La Araucanía. Finalmente están las regiones que representan una fracción menor, entre el 1,9\% y un 0,3\% del total de vacantes, conformada por Tarapacá, Atacama, Los Ríos, Arica/Parinacota, Magallanes y Aysén.

Tabla n. $^{\circ}$ 2: Datos generales de vacantes por región 2011.

\begin{tabular}{|c|c|c|c|c|}
\hline \multirow[b]{2}{*}{ Región } & \multicolumn{3}{|c|}{ Vacantes por región } & \multirow{2}{*}{$\begin{array}{l}\text { Otros indicadores regionales } \\
\begin{array}{c}\text { Ingreso monetario per cápita } \\
\text { de los hogares (promedio, } \\
\text { en pesos) }\end{array}\end{array}$} \\
\hline & $\begin{array}{c}\text { Total } \\
\text { vacantes }\end{array}$ & $\begin{array}{l}\text { \% Respecto } \\
\text { del país }\end{array}$ & $\begin{array}{c}\text { Tasa vacantes respecto } \\
\text { población de } 20 \text { a } 24 \\
\text { años (a) }\end{array}$ & \\
\hline Arica/Parinacota & 3.667 & 1,2 & 0,24 & 185.339 \\
\hline Tarapacá & 5.946 & 1,9 & 0,22 & 177.399 \\
\hline Antofagasta & 11.491 & 3,7 & 0,23 & 204.535 \\
\hline Atacama & 3.546 & 1,1 & 0,15 & 168.171 \\
\hline Coquimbo & 13.785 & 4,4 & 0,23 & 156.952 \\
\hline Valparaíso & 33.801 & 10,9 & 0,22 & 185.769 \\
\hline Metropolitana & 147.848 & 47,6 & 0,24 & 284.979 \\
\hline O'Higgins & 10.422 & 3,4 & 0,15 & 167.563 \\
\hline Maule & 15.758 & 5,1 & 0,19 & 154.420 \\
\hline Biobío & 34.006 & 10,9 & 0,19 & 152.883 \\
\hline La Araucanía & 13.289 & 4,3 & 0,16 & 134.663 \\
\hline Los Ríos & 3.967 & 1,3 & 0,12 & 140.515 \\
\hline Los Lagos & 9.556 & 3,1 & 0,14 & 170.550 \\
\hline Aysén & 842 & 0,3 & 0,10 & 230.230 \\
\hline Magallanes & 2.834 & 0,9 & 0,22 & 238.121 \\
\hline TOTAL & 310.758 & 100 & 0,21 & 214.019 \\
\hline
\end{tabular}

Fuente: CNED (2011), INE (2012), CASEN (2009).

Nota: (a) Indicador: vacantes/estimación población entre 20 y 24 años de la Región.

Para establecer una comparación válida de las vacantes de regiones, más allá del número absoluto, se utiliza la cobertura de vacantes según la población de entre 20 y 24 años. En razón de ello, se observa que las regiones con menor número de vacantes según su población son: Aysén, Los Ríos y Los Lagos, que cubren entre el $10 \%$ y el 14\% de su población de ese tramo etario. En tanto, las con mejor cobertura son la Metropolitana, Arica/Parinacota, Antofagasta y Coquimbo, que tienen una cobertura entre 23\% y 24\% de su población para el mismo tramo de edad anterior. Cabe destacar las regiones de Magallanes y de Arica/Parinacota, que pese a su condición territorial "extrema" y pertenecer a las regiones con menor número de 

PERSPECTIVA TERRITORIAL: INEQUIDADES Y ASIMETRÍAS EN EL MERCADO - S. Donoso , Ó. Arias, M. Weason, C. Frites

vacantes, corresponden al grupo con mejor cobertura etaria. Un factor que explicaría esta situación es que ambas regiones, situadas en los extremos opuestos del territorio, comparten una alta concentración de la población urbana en una o dos ciudades, lo que implica mayor cobertura del sistema escolar y una tasa de rendimiento razonable en ese nivel. Asimismo, poseen centros de educación universitaria desde hace cinco décadas, que fueron impulsados en sus inicios por razones geopolíticas; finalmente en estos casos el costo/oportunidad de estudiar en otra región es muy alto, lo que incentiva la incorporación de la población local a estos centros de enseñanza.

Los datos sobre el promedio del ingreso per cápita de los hogares (al año 2011) muestran una media nacional de 214019 pesos. Estos registros dan cuenta, en primer lugar, que las regiones con menores ingresos per cápita del hogar -La Araucanía y Los Ríoscoincidentemente tienen una baja tasa de vacantes por población de entre 20 y 24 años $(0,16$ y 0,12 respectivamente). Por otro lado, las regiones con mayores ingresos -Metropolitana, Magallanes, Aysén y Antofagasta- se encuentran en posiciones superiores respecto a este mismo indicador, con la excepción de la Región de Aysén. Situación en alguna medida consistente, ya que los costos de la formación de pregrado son financiados en una proporción importante por los estudiantes y sus familias, de manera que la sustentabilidad de esta formación depende del poder adquisitivo de sus habitantes. Ello explica, por ejemplo, la forma como se fue implementado la expansión de los centros de educación superior en el país, tras la reforma de 1981, apreciándose que primero se instalaron en los centros regionales de mayor dinamismo y tradición, para posteriormente descalzarse a las ciudades intermedias y posteriormente a las más pequeñas, proceso que igualmente ha seguido el sentido inverso cuando no ha sido exitoso (Donoso, 2010).

La Región de Aysén, como indicamos, se aleja de la tendencia señalada, aunque teniendo ingresos económicos per cápita por sobre la media nacional, presenta la cobertura más baja para su población entre 20 y 24 años y el menor número de vacantes en el país, situación asociada probablemente a sus problemas de accesibilidad territorial, las distancias entre las localidades, razones climáticas y baja densidad 
poblacional absoluta y relativa, de allí que la oferta sea escasa y no contemple carreras profesionales más tradicionales cuyo nivel de exigencia de formación es mucho más alto.

Tabla n. ${ }^{0}$ 3. Porcentaje de vacantes 2011 por tipo de institución educacional (\% por región).

\begin{tabular}{|l|c|c|c|c|c|c|c|r|}
\hline Región & CFT & IP & $\begin{array}{r}\text { UES } \\
\text { estatales }\end{array}$ & $\begin{array}{c}\text { UES priv. } \\
\text { tradicionales }\end{array}$ & $\begin{array}{c}\text { UES priv. no } \\
\text { tradicionales }\end{array}$ & $\begin{array}{c}\text { Total } \\
\text { universidades }\end{array}$ & Total & $\begin{array}{c}\text { Vacantes } \\
2011\end{array}$ \\
\hline Arica/Parinacota & 27,0 & 8,6 & 45,5 & 0,0 & 18,9 & 64,4 & 100,0 & 3.667 \\
\hline Tarapacá & 19,1 & 11,3 & 35,8 & 0,0 & 33,8 & 69,6 & 100,0 & 5.946 \\
\hline Antofagasta & 18,4 & 37,2 & 19,3 & 11,1 & 14,0 & 44,4 & 100,0 & 11.491 \\
\hline Atacama & 18,3 & 36,9 & 23,0 & 0,0 & 21,8 & 44,8 & 100,0 & 3.546 \\
\hline Coquimbo & 19,5 & 43,5 & 11,2 & 4,4 & 21,5 & 37,0 & 100,0 & 13.785 \\
\hline Valparaíso & 16,0 & 34,9 & 12,0 & 15,7 & 21,4 & 49,1 & 100,0 & 33.801 \\
\hline Metropolitana & 20,3 & 43,9 & 6,8 & 3,3 & 25,7 & 35,8 & 100,0 & 147.848 \\
\hline O'Higgins & 16,7 & 74,8 & 0,3 & 0,0 & 8,2 & 8,5 & 100,0 & 10.422 \\
\hline Maule & 12,7 & 51,0 & 9,9 & 7,7 & 18,6 & 36,3 & 100,0 & 15.758 \\
\hline Biobío & 14,7 & 46,2 & 6,5 & 17,1 & 15,5 & 39,1 & 100,0 & 34.006 \\
\hline La Araucanía & 27,2 & 29,7 & 16,0 & 14,4 & 12,8 & 43,2 & 100,0 & 13.289 \\
\hline Los Ríos & 22,1 & 16,9 & 0,0 & 48,8 & 12,2 & 61,0 & 100,0 & 3.967 \\
\hline Los Lagos & 16,2 & 57,7 & 8,9 & 4,3 & 12,9 & 26,1 & 100,0 & 9.556 \\
\hline Aysén & 38,8 & 53,4 & 0,0 & 0,0 & 7,7 & 7,7 & 100,0 & 842 \\
\hline Magallanes & 27,5 & 19,6 & 29,1 & 0,0 & 23,8 & 52,9 & 100,0 & 2.834 \\
\hline TOTAL & 19,0 & 42,4 & 9,7 & 7,5 & 21,4 & 38,6 & 100,0 & 310.758 \\
\hline
\end{tabular}

Fuente: CNED (2011).

Por su parte, en el país la oferta en educación superior para el año 2011 es provista desde el punto de vista cuantitativo mayormente por los IP, que explican el 42,4\% del total, seguida por las universidades privadas no tradicionales responsables del 21,4\%, luego por los CFT con el 19\% de las vacantes. Las universidades públicas (estatales) dan cuenta del 9,7\% de las vacantes y las universidades privadas tradicionales del $7,5 \%$.

La distribución de las vacantes en el país según tipo de institución tiende a ser diferente de lo que acontece en cada región, no obstante en las regiones Metropolitana, de Coquimbo, Valparaíso y del Maule hay mucha semejanza en esta dimensión con la nacional; el peso estadístico de las vacantes en la Región Metropolitana determina en gran parte los resultados del país, perdiéndose la representatividad de la realidad de otras comunas. Si bien en once de las quince regiones la mayor oferta es generada por IP, en las regiones de Tarapacá, 

PERSPECTIVA TERRITORIAL: INEQUIDADES Y ASIMETRÍAS EN EL MERCADO - S. Donoso, Ó. Arias, M. Weason, C. Frites

Magallanes y Arica/Parinacota son las universidades públicas las que tienen mayor presencia, mientras que en la Región de Los Ríos, donde no existen universidades estatales, es una universidad privada tradicional -Austral de Chile- la que dispone del mayor número de vacantes.

En siete regiones el segundo lugar en vacantes lo registran los CFT y en seis las universidades privadas no tradicionales. Una situación que se presenta en la mayoría de las regiones -doce de ellas-, es que las instituciones con el número más bajo de vacantes son las universidades privadas tradicionales.

Mientras todas la regiones cuentan con CFT, IP y universidades privadas no tradicionales, solamente una no dispone de universidades públicas (Los Ríos) y nueve poseen universidades privadas tradicionales (no tienen Tarapacá, Atacama, O’Higgins, Aysén, Magallanes y Arica/Parinacota).

Si bien en todas las regiones se observa una marcada tendencia en materia de oferta de vacantes hacia la primacía de los IP y CFT, hay diferencias en la oferta de las instituciones universitarias. Mientras el promedio nacional de las vacantes provistas por estas entidades es de 38,6\%, aproximadamente la mitad de las regiones tienen una cifra similar, cuatro regiones registran por sobre el $50 \%$ de sus vacantes en estas instituciones (Tarapacá, Arica/Parinacota, Los Ríos y Magallanes) y en el otro extremo dos regiones alcanzan menos del 10\% de sus vacantes en universidades (Aysén y O'Higgins), con razones muy diferentes en cada caso, como ha sido expuesto con antelación.

Al analizar la distribución de las vacantes según área del conocimiento, el área con mayor presencia es Tecnología (85 706 - 27,6\% del total), seguida de: Administración y Comercio, Salud y Educación, y luego por Ciencias Sociales y Arte y Arquitectura. Hay áreas con muy poca representación en el total de vacantes: Derecho, Recursos Naturales, Ciencias y Humanidades. En cierta manera esta distribución tiene cierta semejanza con la forma como se distribuye la educación técnico-profesional (de enseñanza secundaria) en el país, donde las áreas dominantes son las mismas tres primeras, solo que con un orden diferente (Mineduc, 2012). 


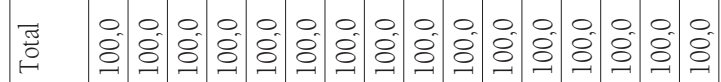

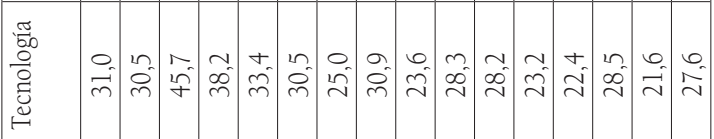

吾 吾

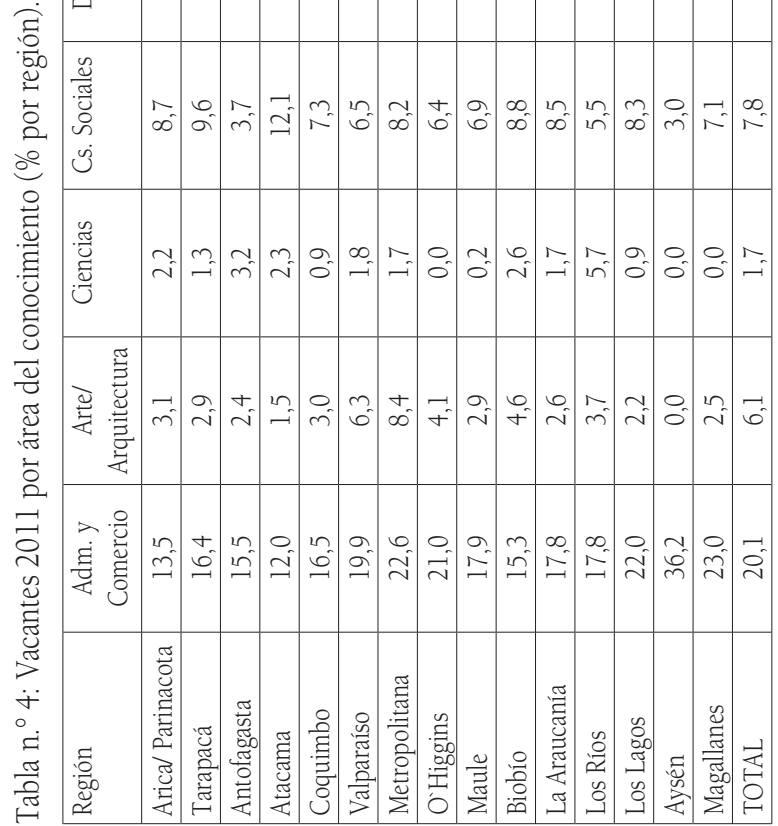

衣 

PERSPECTIVA TERRITORIAL: INEQUIDADES Y ASIMETRÍAS EN EL MERCADO - S. Donoso, Ó. Arias, M. Weason, C. Frites

Esta distribución se reproduce de manera similar en las regiones. En trece de ellas el área del conocimiento con mayor número de vacantes es Tecnología. En las dos regiones donde esto no ocurre, Aysén y Magallanes, es Administración y Comercio la de mayor oferta. En catorce regiones las cuatro áreas de conocimiento con mayor presencia son Tecnología, Administración y Comercio, Salud y Educación, aunque no necesariamente en ese orden.

En tanto, respecto de las áreas con menor número de vacantes, la situación difiere parcialmente de los resultados nacionales. Humanidades y Ciencias son las áreas con menor presencia en ambos planos. La primera, Humanidades, en todas las regiones se encuentra dentro de las tres con menor número de vacantes, y en nueve es la con menos vacantes. Ciencias en doce regiones está dentro de las tres áreas del conocimiento con menor oferta de vacantes. En tercer lugar Arte y Arquitectura en nueve regiones se encuentran en este grupo de las tres áreas con menor presencia.

Complementariamente, en once regiones hay vacantes en todas las áreas de conocimiento. A excepción de Magallanes y Los Ríos que no disponen oferta en Ciencias y Humanidades respectivamente. Por su parte, la Región de O'Higgins no tiene vacantes en dos áreas, Ciencias y Humanidades, y Aysén no cuenta con oferta en Arte y Arquitectura, Ciencias, Derecho y Humanidades.

En relación con el tipo de carrera impartida, el número de vacantes nacionales está liderado por las técnicas de nivel superior, con 136923 que representan el 44,1\% del total. Seguidas por las carreras profesionales con licenciatura que tuvieron 91448 vacantes, equivalentes al 29,4\% del total, y en tercer lugar las carreras profesionales (que no otorgan licenciatura) con 74882 vacantes, correspondiendo al 24,1\%. Los otros tipos de carrera existentes, bachillerato, licenciatura plan común o ciclo básico, ofrecen un número reducido de vacantes, representando entre el $0,6 \%$ y $1 \%$ del total. 


\section{Clasificación de las regiones en función de la oferta de educación superior}

Con la finalidad de alcanzar una mejor comprensión de la oferta territorial de vacantes de las diversas instituciones de educación superior se ha generado una tipología de cinco grupos, en consideración de criterios como número de vacantes, diversidad y nivel de formación ofertado. La tipología no solamente posee finalidades analíticas, sino que también representa a objetos de naturaleza diversa, es decir diferenciables bajo atributos o características relevantes, más aún en términos de la implantación de políticas de formación de capital humano $(\mathrm{KH})$ asociadas al desarrollo de los territorios. En la tabla n. ${ }^{0} 5$ se exponen los valores registrados en los diversos componentes para cada una de las regiones. 
112 LA OFERTA DE EDUCACIÓN SUPERIOR DE PREGRADO EN CHILE DESDE LA PERSPECTIVA TERRITORIAL: INEQUIDADES Y ASIMETRÍAS EN EL MERCADO - S. Donoso , Ó. Arias, M. Weason, C. Frites

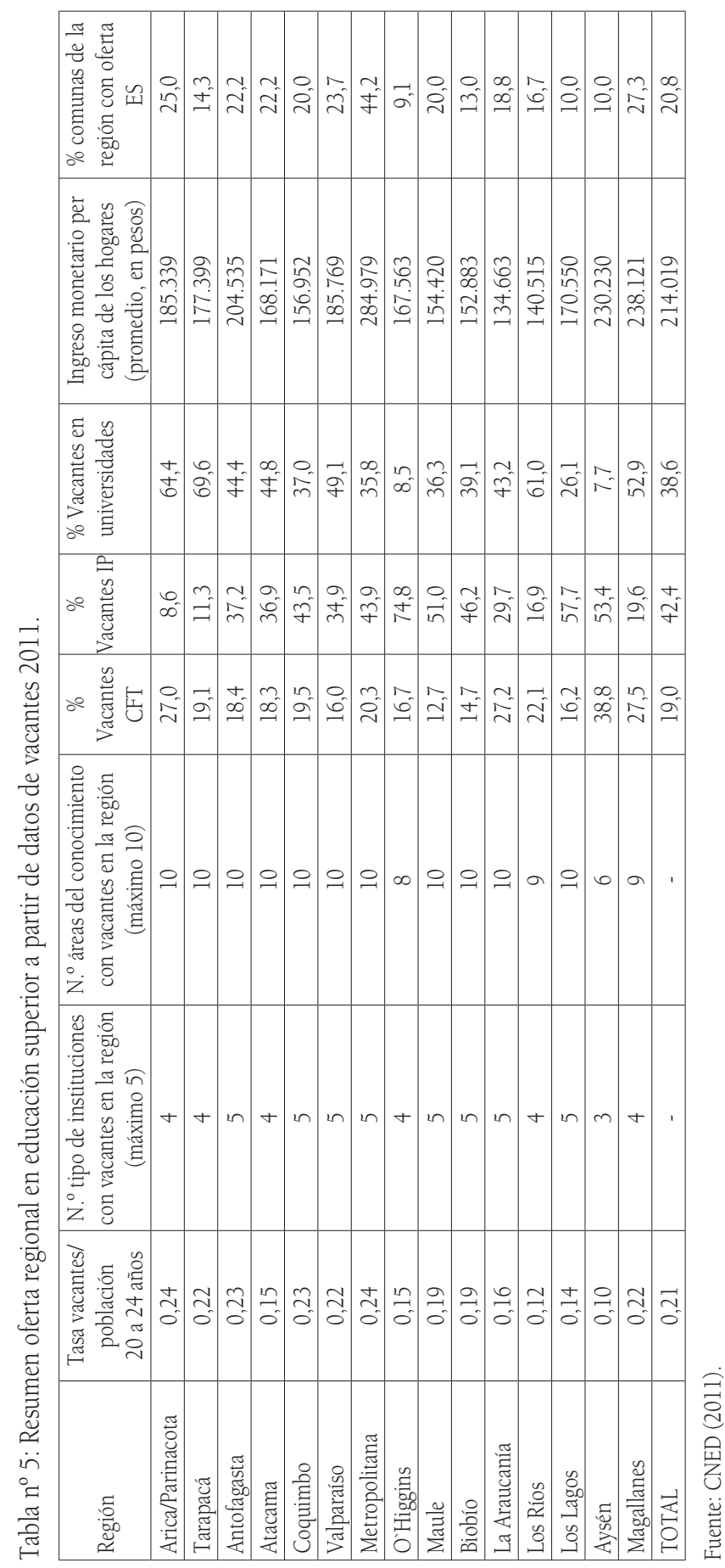


Los grupos constituidos se caracterizan por los siguientes atributos:

Primer grupo: Región Metropolitana.

(a) La región concentra la oferta de educación superior 2011, con el 32\% de las comunas del país y el 48\% de las vacantes, (b) Posee una oferta completa y diversa, con presencia de todos los tipos de instituciones (19 CFT, 26 IP y 32 universidades), y (c) programas académicos en todas las áreas del conocimiento, (d) Es la región con mayor proporción de oferta respecto a su población entre 20 y 24 años y el ingreso per cápita por hogar más alto del país.

Segundo grupo: Regiones de Valparaíso y Biobío

(a) Cuentan con oferta en todos los tipos de instituciones de educación superior y con programas académicos en todas las áreas del conocimiento. Ambas regiones se constituyen -respectivamente- como uno de los principales centros en educación terciaria, (b) no obstante se registran algunas diferencias que posicionan en una condición relativamente mejor a la Región de Valparaíso, pues reúne un mayor porcentaje de vacantes en instituciones universitarias (la más alta en las regiones con alto número de vacantes) y, respecto a tasa de vacantes por población (de 20 a 24 años), la Región de Valparaíso, a diferencia de la de Biobío, está en la mitad superior de la distribución y por sobre la media nacional, (c) Ambas regiones tienen ingresos per cápita de los hogares bajo la media nacional.

Tercer grupo: regiones de Antofagasta, Coquimbo, Maule, La Araucanía, O'Higgins y Los Lagos

(a) Estas regiones registran entre un 3,1\% del total (Los Lagos) y un 5,1\%, del total (Región del Maule), y (b) entre 6 y 2 comunas con oferta en educación superior. Respecto a la diversidad de su oferta, (c) reúnen todos los tipos de instituciones y programas en todas las áreas del conocimiento, exceptuando la Región de O'Higgins, 
que no tiene universidades privadas tradicionales y posee solo ocho áreas del conocimiento, (d) Acerca de los otros indicadores, estas regiones no tienen una tendencia clara: entre 0,14 y 0,23 en la tasa de vacantes 2011 por población entre 20 y 24 años. El porcentaje de vacantes en universidades es entre 8,5\% (en O'Higgins) y $44,4 \%$ (en Antofagasta). Los ingresos de estas regiones están por debajo de la media nacional, con la salvedad de Antofagasta.

Cuarto grupo: regiones de Los Ríos, Tarapacá, Atacama y Arica y Parinacota.

Estas regiones se caracterizan por (a) una baja cantidad de vacantes, menor al $2 \%$ nacional y tienen como máximo dos comunas con oferta en ese nivel, (b) A pesar del menor volumen, presentan cierta diversidad en su oferta con al menos cuatro tipos de instituciones, nueve áreas del conocimiento y tres tipos de carrera, (c) La situación respecto a la tasa de vacantes por población entre 20 y 24 años es más diversa, mientras en Los Ríos y Atacama tienen una baja oferta para este grupo etario, de 12 y $15 \%$ respectivamente; Arica/Parinacota y Tarapacá muestran mejores resultados con tasas de 24 y $22 \%$ respectivamente. Es posible observar que registran una alta proporción de vacantes en instituciones universitarias, e ingresos per cápita promedio de los hogares entre 140 mil y 185 mil pesos.

Quinto grupo: regiones de Aysén y Magallanes

(a) Ambas regiones del extremo sur del país tienen un bajísimo número de vacantes, menor al 1\% del total nacional. Sin embargo, (b) registran diferencias importantes en la caracterización de su oferta, encontrándose Magallanes en mejor situación. Esta región posee alrededor de la mitad de su oferta en instituciones universitarias, tiene cuatro de los tipos de instituciones educacionales (no tiene universidades privadas tradicionales) y nueve de las diez áreas del conocimiento (no cuenta con programas en el área de Ciencias). Aysén tampoco cuenta con universidades 
privadas tradicionales, tiene solo seis áreas del conocimiento y la cobertura para su población entre 20 y 24 años es la más baja del país, (c) Los ingresos promedio de los hogares de ambas regiones son muy similares y están por sobre la media nacional.

Esta clasificación retrata la oferta heterogénea de educación superior en el país, adscrita a un conjunto de inequidades territoriales de larga data, cuya corrección ha sido muy lenta, pese a la ampliación de las vacantes mediante el mercado; se aprecia aún que hay muchos territorios con educación precaria (en algunas áreas y en formaciones de nivel técnico a lo más), lo que da cuenta de la necesidad de insertar correctores de equidad territorial relevantes en este campo, que tengan por objetivo, precisamente, contribuir a una mayor equidad en las oportunidades de la sociedad (Roemer, 2000), porque pareciera ser que el mercado no es capaz de regular estos procesos que implican resultados de mediano y largo plazo, cuyas rentabilidades son lentas y no siempre se expresan monetariamente con tasas de retorno altas ni ajustadas a las expectativas, no obstante ello no implica que deba excluirse de oportunidades en esta materia a ese territorio y su población, medidas a las cuales - por cierto- el mercado no atiende ni prevé

\section{Distribución de la oferta regional}

Al analizar la distribución de oferta de educación superior en cada región, se observan diferencias importantes entre comunas. A partir de la clasificación presentada en la sección anterior es posible señalar lo siguiente:

a. La Región Metropolitana tiene el mayor número de comunas con esta oferta educativa, 23 de las 72 del país. De ellas, 21 se localizan en el Gran Santiago, y solamente dos en la zona suburbana.

5 No debe confundirse este aspecto con políticas públicas específicas para casos extremos, donde el mercado no opera. La relación educación superior, políticas de desarrollo del país y formación de $\mathrm{KH}$ avanzado son cuestiones para las cuales el mercado no ha dado respuestas eficientes y se requiere de políticas de mediano largo plazo cuya sustentabilidad no puede regirse por cuestiones de corto plazo. 
- La realidad de la oferta de las comunas del Gran Santiago es muy diversa. Existen comunas con un propuesta muy básica y escasos cupos: La Reina, Quilicura o La Pintana, cuyas vacantes el año 2011 no representan más allá del 1,5\% del total nacional, con una sola institución. Mientras que en el otro extremo, la comuna de Santiago registra el 28\% del total de vacantes 2011 del país, disponiendo de 52 instituciones (14 CFT, 19 IP, y 19 universidades).

- También otras comunas disponen de un nivel muy alto de ofertas. Providencia (4\% del total nacional de vacantes), con 17 instituciones (en todas las categorías), y Las Condes (1,97\% de la oferta nacional 2011 y 7 instituciones).

- A pesar de la gran diferencia entre las comunas presentes en el Gran Santiago, su locación al interior de la ciudad hace que se pueda comprender como una misma oferta con un gran volumen y diversidad, tanto de programas como de instituciones.

- La zona suburbana, en tanto, solo posee oferta en las comunas de Buin y Melipilla. En ambos casos es muy acotada en volumen, y tiene entre 2 y 4 instituciones. Buin no cuenta con instituciones universitarias, y Melipilla dispone de una universidad privada no tradicional.

b. Las regiones de Valparaíso y Biobío tienen 9 y 7 comunas con educación superior, respectivamente. Ambas registran dos comunas con importante oferta, Viña del Mar y Valparaíso en la primera, y Concepción y Chillán en la segunda. Estas cuatro comunas tienen entre 10 y 21 instituciones (incluidos CFT, IP y universidades), y programas en las 10 áreas del conocimiento. Biobío también tiene la comuna de Los Ángeles con una oferta relevante y con diversas alternativas. El resto de sus comunas tienen una participación menor, casi sin oferta universitaria.

c. Las regiones de Antofagasta, Coquimbo, Maule, La Araucanía, O'Higgins y Los Lagos tienen de 2 a 6 comunas con oferta de educación superior. Entre una y dos comunas con una mayor presencia, que tienen universidades y con 9 o 10 áreas del conocimiento, y entre 10 y 17 instituciones. 
d. Las regiones de Los Ríos, Tarapacá, Atacama y Arica y Parinacota poseen entre 1 y 2 comunas con oferta, solo una de mayor importancia con universidades, entre 8 y 10 instituciones de educación superior, y un volumen de vacantes entre 1 y el 1,9\% de total nacional 2011.

e. La región de Aysén cuenta con solo una comuna con educación superior, Coyhaique con 4 instituciones y solo una universidad (privada no tradicional). La región de Magallanes en tanto cuenta con tres comunas. De ellas Porvenir y Puerto Natales tienen solo una universidad pública, y Punta Arenas 8 instituciones, entre estas CFT, IP, una universidad pública y dos universidades privadas no tradicionales. Aun cuando hay presencia de instituciones, la cantidad de matrículas ofrecidas el año 2011 en las comunas de ambas regiones es muy baja, no superando el 1\% del total nacional.

La descripción anterior plantea una distinción en el tipo de oferta en educación superior con que cuentan las comunas, que puede ser simple o compleja ${ }^{6}$ de acuerdo a las características de la comuna (tablas n. ${ }^{\circ} 7$ y n. ${ }^{\circ} 8$ ), en reconocimiento a las distintas escalas territoriales (SUBDERE, 2011). Para la elaboración de esta clasificación se utilizaron los siguientes indicadores: número de instituciones en la comuna, porcentaje de vacantes del país, áreas del conocimiento, presencia de universidades, presencia de universidades pertenecientes al Consejo de Rectores de las Universidades Chilenas, CRUCH, y tipo de instituciones presentes en la comuna (CFT, IP, universidades públicas, universidades privadas tradicionales y universidades privadas no tradicionales).

A partir de esta clasificación se obtiene que 22 de las 72 comunas con educación superior en el país (33\%) poseen oferta compleja, el restante $66,7 \%$ (50 comunas) es de tipo simple. Por otro lado, se establece que en varias comunas la oferta provista es complementada por la oferta de comunas contiguas, debido a la factibilidad de traslado que tienen sus habitantes. En la práctica, las alternativas en estudios

6 Detalle de indicadores en anexo 1. 

PERSPECTIVA TERRITORIAL: INEQUIDADES Y ASIMETRÍAS EN EL MERCADO - S. Donoso, Ó. Arias, M. Weason, C. Frites

superiores se incrementan para los estudiantes de dichas comunas por esta situación. Al considerar estos dos criterios de clasificación: (i) comunas con oferta simple o compleja, y (ii) comunas contiguas con oferta o comunas aisladas (no contiguas a otras comunas con oferta), se obtiene el siguiente resultado ${ }^{7}$.

Tabla n. ${ }^{\circ}$ 6. Clasificación de las comunas con presencia de educación superior, según proximidad territorial y complejidad de la oferta.

\begin{tabular}{|l|c|c|}
\hline & Simple & Compleja \\
\hline Contigua & 17 & 8 \\
\hline Aislada & 13 & 11 \\
\hline
\end{tabular}

Fuente: Elaboración propia.

Sobre la base de la caracterización de la tabla n. ${ }^{\circ} 7$, se puede plantear la existencia de "territorios de educación superior", es decir, espacios de comunas adyacentes donde, debido a su cercanía territorial y posibilidad de traslado o movilidad entre ellas, es posible considerar una oferta conjunta.

Tabla n. ${ }^{\circ}$ 7. Caracterización de comunas con presencia de ES según tipo de oferta y características territoriales.

\begin{tabular}{|c|c|} 
Macroterritorios con oferta compleja \\
\hline Región & Comunas \\
\hline IV & La Serena \\
\hline V & Valparaíso \\
\hline V & Viña del Mar \\
\hline VI & Rancagua \\
\hline VIII & Concepción \\
\hline IX & Temuco \\
\hline X & Puerto Montt \\
\hline XII & Punta Arenas \\
\hline
\end{tabular}

Territorios aislados con oferta compleja

\begin{tabular}{|c|c|}
\hline Región & Comunas \\
\hline I & Iquique \\
\hline II & Antofagasta \\
\hline II & Calama \\
\hline III & Copiapó \\
\hline VII & Curicó \\
\hline VII & Talca \\
\hline VIII & Chillán \\
\hline VIII & Los Ángeles \\
\hline X & Osorno \\
\hline XIV & Valdivia \\
\hline XV & Arica \\
\hline
\end{tabular}

7 Se excluyeron las comunas de la Región Metropolitana por la alta concentración de la oferta de educación superior, que implica una situación muy diferente respecto de las otras regiones. Como se señaló, esta región tiene 23 comunas con oferta, 21 de ellas en el Gran Santiago, lo que configura un gran macroterritorio que no resulta pertinente analizar desde las categorías de comunas aisladas o contiguas. 
Territorios contiguos con oferta simple

\begin{tabular}{|c|c|}
\hline Región & Comunas \\
\hline IV & Coquimbo \\
\hline IV & Ovalle \\
\hline V & Quilpué \\
\hline V & San Felipe \\
\hline V & Los Andes \\
\hline V & Quillota \\
\hline V & La Calera \\
\hline VI & Graneros \\
\hline VII & Parral \\
\hline VII & Cauquenes \\
\hline VIII & Talcahuano \\
\hline VIII & Lebu \\
\hline VIII & Arauco \\
\hline VIII & Lota \\
\hline IX & Vilcún \\
\hline X & Puerto Varas \\
\hline XII & Porvenir \\
\hline
\end{tabular}

Fuente: Elaboración propia.
Territorios aislados con oferta simple

\begin{tabular}{|c|c|}
\hline Región & Comunas \\
\hline III & Vallenar \\
\hline V & La Ligua \\
\hline V & San Antonio \\
\hline VI & San Fernando \\
\hline VII & Constitución \\
\hline VII & Linares \\
\hline IX & Angol \\
\hline IX & Curarrehue \\
\hline IX & Victoria \\
\hline IX & Villarrica \\
\hline XI & Coyhaique \\
\hline XII & Puerto Natales \\
\hline XIV & Río Bueno \\
\hline
\end{tabular}

Los cuatro tipos de territorios se caracterizan por los siguientes aspectos:

1. Macroterritorios con oferta compleja: Representan comunas con alta diversidad en su oferta en educación superior con otras de menor diversidad, conformando en su interior una oferta más completa. Tres elementos son claves en su identificación, (1) la densidad poblacional del territorio en el que se insertan, que permita dar sustento a una oferta de educación variada; (2) la proximidad con otros enclaves urbanos de cierta significación, y (3) la tasa de ruralidad y dispersión de su población en el territorio. En razón de ello, el grupo está liderado por los enclaves de mayor concentración de población entre La Serena y Rancagua, y luego Temuco, Puerto Montt y Punta Arenas; considerando ciertamente a la Región Metropolitana.

Estos territorios, generados en forma mixta a partir de las universidades tradicionales, fueron los primeros centros donde se instalaron instituciones privadas pos reforma de 1981, también su oferta es completa y a veces duplicada por diversas instituciones, lo que implica ineficiencia social. Su principal característica es 
que están rodeadas de aquellas comunas del tercer grupo, que precisamente dan sustento a la densidad poblacional requerida para hacer viables estas propuestas formativas.

2. Territorios aislados con oferta compleja: Se trata de comunas que poseen cierta densidad poblacional que permite ofertas complejas, amparadas además en distancias relevantes con otras unidades territoriales de alta densidad, como corresponde al norte grande del país, o bien son comunas capitales de región y/o provincia, rodeadas de comunas rurales con importante número de habitantes. En el primer subgrupo de esta categoría están Arica, Iquique, Antofagasta, Calama, Copiapó. Territorios fuertemente limitados por su emplazamiento geográfico para obtener mayor masa estudiantil. Mientras que en el segundo subgrupo están las comunas de Curicó, Talca, Chillán, Los Ángeles, Osorno, Valdivia y Arica, que por razones de concentración de la población urbana, distancia territorial o por estar rodeados de comunas de alta ruralidad, no conforman unidades de densidad poblacional suficientes para transformarse en enclaves en esta materia.

El primer subgrupo de comunas requiere de una política de apoyo, en razón de sus características territoriales, muy diferentes del segundo subgrupo, que puede tener mayor autosustentabilidad porque la densidad poblacional es mayor.

3. Territorios contiguos con oferta simple: Podrían ser comprendidos también como una subcategoría del primer grupo, por cuanto su existencia está principalmente asociada a los enclaves urbanos superiores del país, salvo algunas excepciones. En esta situación están en la región de Coquimbo, el territorio conformado por las comunas de Coquimbo, La Serena y Ovalle. En la región de Valparaíso, el compuesto por las comunas de Valparaíso, Viña del Mar y Quilpué. En la región de O'Higgins, el territorio de Rancagua y Graneros; y en Biobío, ciertamente el territorio de las comunas de Concepción y Talcahuano.

Las políticas públicas en esta materia -por la funcionalidad que prestan estas comunas- deberían desprenderse de políticas de los macroterritorios, no obstante se requiere de precisiones que se traduzcan en apoyo para todas y no solamente para las cabeceras de los macroterritorios, es decir han de implementarse políticas 
públicas para "que todos ganen" en una perspectiva de mediano y largo plazo.

4. Territorios aislados con oferta simple: Agrupan comunas que poseen menor diversidad en su oferta de educación superior con otras, mejorando en su conjunto la oferta individual. También se puede identificar en este segmento a la Región de Valparaíso, pero con dos territorios. El primero conformado por San Felipe y Los Andes, y el segundo por Quillota y La Calera. De igual manera la Región del Biobío dispone de un territorio adicional compuesto por las comunas de Lebu, Arauco y Lota.

Las regiones de Valparaíso y del Biobío, ambas con una gran oferta en educación superior, poseen los dos tipos de territorios educacionales. Mientras la mayoría de las regiones están conformadas por un territorio de menor oferta y otros centros aislados. Las políticas en este caso debiesen ir por el lado de generar redes que permitirán, al cabo de unos años de estudio, el traslado de aquellos estudiantes que buscan una oferta diferente, al menor costo posible para ellos, es decir bajo sistemas de bachillerato $u$ otras modalidades, con un sistema de soporte financiero y académico para minimizar los impactos en este plano.

\section{Elementos para el debate de políticas públicas para el desarrollo territorial de la educación superior en Chile}

La hipótesis que sustenta este trabajo se confirma (no se rechaza): la oferta de educación superior en Chile se distribuye de manera desigual entre regiones, como igualmente presenta la misma característica intrarregión en las comunas, debido a la desigualdad socioeconómica y territorial que caracteriza al país y sus impactos sobre la densidad poblacional, elementos que apuntan en lo fundamental a uno de los problemas más complejos a que debe contribuir la educación, la justicia distributiva. Cuestión que en este caso no se cumple en los términos esperados, si bien una retrospectiva histórica de los últimos treinta años muestra un panorama mucho más equitativo, esto no implica que no deban corregirse aquellas inequidades más relevantes en este ámbito. 

PERSPECTIVA TERRITORIAL: INEQUIDADES Y ASIMETRÍAS EN EL MERCADO - S. Donoso , Ó. Arias, M. Weason, C. Frites

A partir de la división político-administrativa definida por regiones y subsecuentemente comunas, e independiente de la lógica que le subyace -positiva, negativa o neutra-, es a partir de estos niveles territoriales/administrativos que se ha implementado la mayor parte de las políticas educativas en el país, esencialmente en los niveles primario y secundario, y en menor medida en la educación superior, no obstante en este último caso confluyen otros factores que han hecho más compleja su sustentación, pues en el caso chileno, debido a que el pregrado es pagado en gran medida por las familias, se requiere del componente "posibilidades de pago" de la población para sustentar las opciones formativas. Ello explica la dinámica de distribución territorial, como también la oferta de carreras, que no siempre responde a criterios del entorno sino a demandas que exceden el territorio o que sencillamente no se asocian más que a los convencionalismos de las profesiones liberales.

En razón de ello, atendiendo a esta estructura del territorio y la oferta en su diversidad institucional y considerando las distintas áreas del conocimiento que aborda, pareciera que debido a que la expansión de la cobertura y matrícula ha sido implementada principalmente por las instituciones privadas, ha imperado una visión de mercado más que una lógica vinculada con las necesidades del entorno y la generación de aprendizajes en coherencia con una apuesta de desarrollo territorial.

Es importante que se reflexione en el país acerca de la sustentabilidad del desarrollo territorial desde la formación de capital humano inicial y avanzado, cuestión que implica analizar la función de la educación superior en este escenario y definir el papel del mercado, que al tenor de lo expuesto se ha mostrado ineficiente en esta tarea, con un costo directo e indirecto muy importante para las personas y la sociedad, o bien que se propongan en forma paulatina políticas de regulación y ordenamiento de la educación postsecundaria con las propuestas de desarrollo de los diversos territorios, jerarquizados en función de una visión proactiva y no pasiva como en la actualidad se presenta en el caso examinado.

El estudio establece una clasificación de regiones, de acuerdo a su incidencia, la diversidad y complejidad de su oferta, y en relación 
con las comunas, a su propia oferta de educación superior -sea simple o compleja-y la de los municipios vecinos, que establece que una comuna sea definida como aislada o contigua. Clasificaciones esenciales que permiten caracterizar con claridad ciertos patrones y comportamientos comunes a grandes ciudades, como asimismo a las ciudades intermedias y a las pequeñas, acorde las distintas escalas territoriales.

En la primera categoría se consideran tres conurbaciones claves: el Gran Santiago, Valparaíso-Viña y Concepción. Es importante señalar que solo en estos territorios se puede hablar propiamente tal de la existencia de un mercado de educación superior. Su oferta es completa, al alero de una importante demanda que se sustenta en una densidad poblacional local, siendo polos de atracción para la migración de estudiantes esencialmente por la diversidad de su oferta en educación postsecundaria. Como tal, las políticas de estos macroterritorios han de contemplar criterios matriciales que incluyan a las comunas de su entorno -abastecedoras de estudiantes- y que desarrollan ofertas simples. En tal sentido no pueden ser consideradas solamente como proveedoras de potenciales estudiantes, sino que han de plantearse también políticas de apoyo a la calidad de su formación, con sistemas de redes y soporte financiero para ellos, además de encadenamientos verticales (articulaciones) que les permitan progresar desde diversas tipos de formación inicial.

Las ciudades intermedias corresponden principalmente a las capitales regionales o provinciales en regiones centrales y las capitales regionales en las regiones extremas. La oferta de educación superior en estas comunas es importante, pero no alcanza los niveles de diversidad de las grandes ciudades, debido a una menor demanda, que es estructural a estos territorios. Por esta razón, es difícil que en algún momento se logre una diversidad semejante, en tipos de oferta y calidad, a las ciudades de mayor tamaño, lo cual implica que debería generarse algún tipo de políticas compensatorias hacia estos territorios en relación con la provisión de profesionales de carácter estratégico para el desarrollo social y económico. O bien que esta formación sea subsidiada bajo un criterio no mercantil, lo cual requiere "salir del mercado", algo que en la agenda del poder dominante del país parece menos deseable. 
Por su parte, las ciudades pequeñas corresponden a capitales comunales de menor tamaño que las ciudades intermedias, en donde especialmente en los últimos años se ha instalado una oferta incipiente de educación superior, posiblemente por la saturación de los mercados mayores. Debido a las escasas postulaciones los programas ofrecidos, muy específicos y de calidad difícil de precisar, son impartidos por centros de educación esencialmente docentes, que asumen en muchos casos la forma de capacitación o actualización laboral.

Si bien en el trabajo se relaciona el territorio y la oferta de educación superior, aquí no hay alusión directa a la calidad y otros indicadores igualmente importantes -selectividad, acreditación, producción académica, entre otros-, queda pendiente entonces saber qué tipos de estudiantes atraen y el tipo de oferta que produce este efecto.

Una cuestión para resolver en el diseño de una política pública -como se señaló- es determinar si el mercado operará adecuadamente para satisfacer los objetivos de la sociedad en este ámbito. La discusión en esta materia es determinar si finalmente la educación puede operar como un mercado o cuasimercado territorializado, no obstante la tendencia dominante al respecto es que se requiere de políticas claras, poderosas y bien direccionadas que corrijan asimetrías claves de sustentabilidad de la educación superior en territorios medianos y pequeños, que sin esta mediación difícilmente pueden alcanzar resultados positivos al respecto.

Ciertamente lo anterior se traduce en determinar cómo se puede desarrollar este crecimiento, si se va a realizar guiado por la competencia y la dinámica del mercado, con costos para las familias, o bien hay espacio para una política educativa que racionalice la oferta en conjunto con la iniciativa pública y privada en educación superior, y guarde alguna relación con las estrategias de desarrollo regional. Debido a que hasta ahora en estas comunas existen, en la práctica, ofertas únicas, en donde las primeras instituciones que se instalan copan rápidamente toda la demanda, debe analizarse si esta oferta es un aporte al desarrollo del capital humano de la comuna y la región, o si es preferible tenerla en ciudades intermedias y limitarla en comunas menores. 
Al observar los cuatro tipos de territorios descritos en materia de educación superior, se identifica un patrón de comportamiento en la forma como se ha emplazado desde los centros más poblados a los de menor población. A partir del explosivo crecimiento de los últimos años, es posible hipotetizar que, en la medida que existe una demanda y hogares con ingresos suficientes, aparecen ofertas en los distintos territorios, siempre bajo una lógica de mercado. Estas ofertas, sin embargo, parecen iniciarse con alternativas de menor inversión institucional, o bien subordinadas a los centros de mayor desarrollo, para luego consolidarse. Ello genera un intervalo de tiempo entre la instalación de la oferta y su perfeccionamiento, brecha que es mayor en las zonas donde existe menos competencia. Este periodo de consolidación en gran medida es financiado por el gasto privado. Frente a esta situación, las universidades estatales han sido, en alguna medida, colonizadas en su lógica de acción por el mercado, respondiendo y adaptándose, en mayor o menor medida, con peor o mejor suerte, a estas nuevas condiciones.

Debido a que estos procesos de expansión, adaptación y crecimiento de las instituciones de educación superior y sus programas, como se ha dicho, son financiados principalmente por los hogares, es central desde el punto de vista de la sociedad proteger esta inversión por medio de una regulación, asegurando estándares más elevados, en cuanto a calidad de la oferta y a pertinencia desde la óptica regional a los nuevos programas que se proyecten.

Finalmente, es imprescindible insistir en que las políticas generan muchos efectos indirectos positivos y negativos, por ende su implementación debe hacerse cuidadosamente, con antecedentes sólidos que reduzcan las externalidades negativas. Esta tarea no compete solamente al gobierno central, son los gobiernos subcentrales los que pueden no solamente comprometer recursos, sino en mejor medida orientar el proceso y auditar su desarrollo para que se conduzca adecuadamente. 


\section{Referencias bibliográficas}

BC (2012). PIB Regionalizado. Banco Central de Chile. Obtenida en www. bcentral.cl. Visitado el 15/05/2012.

CASEN (2009). Encuesta Nacional de Caracterización Socioeconómica. Obtenida en http://celade.cepal.org/redatam/paises/chl/mideplanii/ casen2009/Index.html. Visitado el 10/12/2011.

CNED (2011). Evolución de la matrícula de educación superior 1994-2011. Consejo Nacional de Educación, Departamento de Investigaciones e información Pública, Santiago de Chile.

Donoso, S. (2010). Notas sobre el Proceso de Expansión Territorial de las Instituciones de Educación Superior en Chile. Instituto de Investigación y Desarrollo Educacional, Universidad de Talca. Talca.

INE (2008). "Chile. Estimaciones y proyecciones de población por sexo y edad. Total regiones. 1990-2020". Obtenido en: http://www.ine.cl/canales/ chile_estadistico/demografia_y_vitales/proyecciones/MenPrincOK.xls. Visitado el 14/09/2012.

INE (2012). Censo de Población y Vivienda, 2012. Resultados Preliminares. Instituto Nacional de Estadísticas. www.ine.cl Visitado el 10/09/2012.

MINEDUC (2012). Oferta demanda de la educación técnico-profesional y entorno productivo en la VI Región. Ministerio de Educación, Fundación Chile.

Roemer, J.E. (2000). Variantes de la igualdad de oportunidades. En: Fractal (México, D.F.) n. ${ }^{\circ} 16$, año 4, vol. V.

SUBDERE (2011). "Masa crítica de condiciones para el desarrollo de las regiones de Chile. Antecedentes para una discusión incipiente". Compilación de síntesis (editadas) de trabajos desarrollados por la SUBDERE, Santiago de Chile. Obtenido en: http://www.subdere.gov. cl/sites/default/files/documentos/articles-83100_archivo_fuente_0.pdf. Visitado el 10/11/2012.

Recibido: 25/05/2012

Aceptado: 24/09/2012 